\title{
A GALOIS TYPE THEOREM IN VON NEUMANN ALGEBRAS
}

\author{
HISASHI CHODA
}

(Communicated by Palle E. T. Jorgensen)

\begin{abstract}
We shall give a simple proof for a Galois type theorem: Let $\alpha$ be a dual free action of a discrete group $\mathrm{G}$ on a factor $M$. If an automorphism $\theta$ of $M$ leaves the fixed point algebra $M^{\alpha}$ pointwise invariant then there exists a $g_{0} \in G$ with $\theta=\alpha_{g_{0}}$.
\end{abstract}

Since the Galois theory for von Neumann algebras was initiated by M. Nakamura and Z. Takeda in [8], the theory has been developed by several authors $([1,4]$ and see [9] for other references ). Recently, Y. Katayama and M. Takesaki [6] establish the asymptotic Galois correspondence for discrete amenable group action on AFD factors. They prove their main theorm by reducing it to the following:

Theorem A [6, Theorem 3.1]. Let $\alpha$ be a dominant free action of a discrete group $G$ on a properly infinite factor $M$ and $M^{\alpha}$ the fixed point algebra of $M$ under $\alpha$. If an automorphism $\theta$ of $M$ leaves $M^{\alpha}$ pointwise invariant, then there exists $g_{0} \in G$ with $\theta=\alpha_{g_{0}}$.

Ikeshoji [5] shows a theorem of the above type for locally compact group actions on von Neumann algebras. Also, as a theory of this type for discrete groups, in [2], we decided an automorphism fixing the fixed point algebra of a discrete automorphism group.

Let $M$ be a von Neumann algebra, $\mathrm{G}$ a countable discrete group, $\alpha$ an action of $\mathrm{G}$ on $M$, and $M^{\alpha}$ the fixed point algebra of $M$ under the action $\alpha$. We consider a Galois type theorem under the following conditions:

(*) There exists a faithful normal expectation $\varepsilon$ of $\left(M^{\alpha}\right)^{\prime}$ onto $M^{\prime}$, where $C^{\prime}$ is the commutant of an algebra $\mathrm{C}$.

(**) There exists a group $\mathrm{H}$ of automorphisms of $M$ with the following properties (1) and (2):

(1) $\mathrm{H}$ is ergodic on the center of $M$.

(2) $\alpha_{g} h=h \alpha_{g}$ for all $g \in G, h \in H$.

We have proved a Galois type theorem:

Theorem B [2, Corollary 3]. Let $M, G, \alpha$ be as above. Suppose that the conditions $(*)$ and $(* *)$ hold and that the action $\alpha$ is free. If an automorphism

Received by the editors November 27, 1990.

1980 Mathematics Subject Classification (1985 Revision). Primary 46L55.

Key words and phrases. Galois theory, von Neumann algebra, factor, action, expectation. 
$\theta$ of $M$ satisfies the following conditions (1) and (2):

(1) $\theta h=h \theta$ for all $h \in H$,

(2) $\theta(x)=x$ for all $x \in M^{\alpha}$,

then there exists a $g_{0} \in G$ with $\theta=\alpha_{g_{0}}$.

In this paper, we shall show that Theorem $A$ is obtained as an application of Theorem B.

At first, we shall show a lemma:

Lemma. If $\alpha$ is a dual action of a discrete group $G$ on $M$ then there exists a faithful normal expectation $\varepsilon$ of $\left(M^{\alpha}\right)^{\prime}$ onto $M^{\prime}$.

Proof. Since the action $\alpha$ is dual, by [9, Theorem II.2.4, p. 28], there exists a strictly wandering projection $p \in M$ for $\alpha$, i.e., $\left\{\alpha_{t}(p) ; t \in G\right\}$ is a partition of the identity such that $\alpha_{t}(p) \alpha_{s}(p)=0$ for $t \neq s$. We can complete the proof of the lemma in the same method as [3, Proof of Corollary 3] or [7, Example] (also cf. [4]). But we give a proof of the rest for the completeness. Put

$$
\varepsilon(x)=\sum_{t \in G} \alpha_{t}(p) x \alpha_{t}(p) \quad\left(x \in\left(M^{\alpha}\right)^{\prime}\right) .
$$

Then $\varepsilon$ is a faithful normal positive linear mapping. We may assume that $M$ acts on a Hilbert space $\mathrm{H}$ and that there exists a unitary representation $\mathrm{u}(\cdot)$ of $\mathrm{G}$ into $\mathrm{H}$ with $\alpha_{t}=\operatorname{Ad} u(t)$ for all $t \in G$, where, for unitary $u$ preserving $M$ invariant, $\operatorname{Ad} u$ is an automorphism of $M$ induced by $u$. In this situation, the von Neumann algebra $\left(M^{\alpha}\right)^{\prime}$ is generated by $\{u(t) ; t \in G\} \cup M^{\prime}$.

For any $m \in M^{\prime}$ and $s \in G$, we have

$$
\begin{aligned}
\varepsilon(m u(s)) & =\sum_{t \in G} \alpha_{t}(p) m u(s) \alpha_{t}(p) \\
& =m \sum_{t \in G} \alpha_{t}(p) u(s) \alpha_{t}(p)=m \sum_{t \in G} \alpha_{t}(p) \alpha_{s t}(p) u(s) \\
& = \begin{cases}m, & (s=e), \\
0, & (s \neq e),\end{cases}
\end{aligned}
$$

where $e$ is the unit of $\mathrm{G}$.

Therefore, the map $\varepsilon$ is a faithful normal expectation of $\left(M^{\alpha}\right)^{\prime}$ onto $M^{\prime}$.

Theorem. Let $M$ be a von Neumann algebra, $G$ a discrete group and $\alpha$ a dual free action of $G$ on $M$.

Suppose the condition (**) holds.

If an automorphism $\theta$ of $M$ satisfies the following conditions (1) and (2):

(1) $\theta h=h \theta$ for all $h \in H$,

(2) $\theta(x)=x$ for all $x \in M^{\alpha}$,

then there exists a $g_{0} \in G$ with $\theta=\alpha_{g_{0}}$.

Proof. By the lemma, the condition $(*)$ for this situation holds, i.e., there exists a faithful normal expectation of $\left(M^{\alpha}\right)^{\prime}$ onto $M^{\prime}$. Then, by Theorem B, there exists a $g_{0} \in G$ such that $\theta=\alpha_{g_{0}}$.

As a corollary, we have the factor case, which shows Theorem A . 
Corollary. Let $\alpha$ be a dual free action of a discrete group $G$ on a factor $M$.

If an automorphism $\theta$ of $M$ leaves $M^{\alpha}$ pointwise invariant then there exists a $g_{0} \in G$ with $\theta=\alpha_{g_{0}}$.

Proof. Put $\mathrm{H}=\{$ id $\}$, where id is the identity automorphism of $M$. Then $\mathrm{H}$ has properties in the condition $(* *)$. Hence, the corollary follows from the theorem.

\section{ACKNOWLEDGMENT}

The author would like to express his thanks to Prof. Y. Katayama and the referee of this paper for informations on Ikeshoji's paper [5].

\section{REFERENCES}

[1] H. Choda, A Galois correspondence in a von Neumann algebra, Tôhoku Math. J. 30 (1978), 491-504.

[2] _ An automorphism fixing the fixed point algebra of an automorphism group, Math. Japon. 24 (1979), 45-51.

[3] M. Choda, Normal expectations and crossed products of von Neumann algebras, Proc. Japan Acad. 50 (1974), 738-742.

[4] M. Henle, Galois theory of $W^{*}$-algebras, preprint.

[5] K. Ikeshoji, A generalization of Roberts-Tannaka duality theorem, J. Math. Soc. Japan 34 (1982), 55-59.

[6] Y. Katayama and M. Takesaki, Asymptotic Galois correspondence for discrete amenable actions on AFD factors, preprint.

[7] N. J. Munch, Conditional expectations and wandering operators for automorphic group actions, Math. Japon. 34 (1989), 65-72.

[8] M. Nakamura and Z. Takeda, $A$ Galois theory for finite factors, Proc. Japan Acad. 36 (1960), 258-260.

[9] Y. Nakagami and M. Takesaki, Duality for crossed products of von Neumann algebras, Lecture Notes in Math., vol. 731, Springer-Verlag, New York and Berlin, 1979.

Department of Mathematics, Osaka Kyoiku University, Tennoji, Osaka, 543, Japan 